\title{
Hospitalização por coqueluche em crianças no período pré e pós-implantação da vacina dTpa para gestantes
}

\section{Hospitalization due to pertussis in children in the pre- and post- implantation periods of the DTaP vaccine for pregnant women}

Sabrina Dimer da Silva' (D), Frederico Friedrich ${ }^{2}$ (D), Lisiane Tuon Generoso Bitencourt ${ }^{1,3}$ (D), Luciane Bisognin Ceretta ${ }^{3}$ (D) , Jacks Soratto ${ }^{1,4}$ (i)

'Programa de Pós-graduação Multiprofissional em Saúde Coletiva, Universidade do Extremo Sul Catarinense (UNESC) - Criciúma (SC), Brasil.

2Programa de Pós-graduação em Medicina Pediatria e Saúde da Criança, Escola de Medicina (FAMED), Pontifícia Universidade Católica do Rio Grande do Sul (PUCRS) - Porto Alegre (RS), Brasil.

${ }_{3}^{3}$ Programa de Pós-graduação em Medicina e Ciências de Saúde, Universidade do Extremo Sul Catarinense (UNESC) - Criciúma (SC), Brasil.

${ }^{4}$ Programa de Pós-graduação em Enfermagem, Universidade Federal de Santa Catarina (UFSC) - Florianópolis (SC), Brasil.

Como citar: Silva SD, Friedrich F, Bitencourt LTG, Ceretta LB, Soratto J. Hospitalização por coqueluche em crianças no período pré e pós-implantação da vacina dTpa para gestantes. Cad Saúde Colet, 2021;29(3):344-350. https://doi. org/10.1590/1414-462X202129030289

\section{Resumo}

Introdução: A imunização da gestante parece proteger o bebê por meio da transferência passiva de anticorpos e ter um impacto na prevenção da doença em bebês que ainda não iniciaram a vacinação contra coqueluche. Objetivo: Analisar o comportamento dos registros de hospitalizações por coqueluche em crianças menores de 1 ano de idade, conforme a cobertura da vacina dTpa para gestantes. Método:Tratase de um estudo ecológico de caráter observacional e quantitativo dos registros de hospitalizações por coqueluche, em crianças menores de 1 ano de idade, no Sul do Brasil, durante o período de 2007 a 2017. Os dados foram obtidos por meio do Sistema de Informação Hospitalar no DATASUS. Resultados: No período analisado, foram registradas 342.139 hospitalizações por doenças respiratórias em crianças menores de 1 ano, sendo que 3.062 foram por coqueluche. No período pré-vacinal, a média anual foi de 102,2/100.000/ ano e, no período pós-vacinal, foi de 46,8/100.000/ano, representando uma queda de $54,2 \%$ no número de registros, variando de 27,8/100.000/ano em 2007 a 139/100.000/ano em 2013, caindo para 47,9/100.000/ ano em 2017. Conclusão: Houve uma redução no número de registros de hospitalizações por coqueluche após a introdução da vacina dTpa, mesmo com uma cobertura vacinal abaixo de $50 \%$.

Palavras-chave: vacina contra coqueluche; cobertura vacinal; criança.

\begin{abstract}
Background: Immunization of pregnant women seems to protect infants through passive antibody transfer, and have an impact on disease prevention in infants who have not yet started vaccination for pertussis. Objective: Analyze the behavior of hospitalization records for pertussis in children under 1 year of age according to the coverage of the Diphtheria, Tetanus and acellular Pertussis (DTaP) vaccine for pregnant women. Methods: This is an ecological, observational, quantitative study conducted with records of hospitalization due to pertussis in children under 1 year of age in southern Brazil from 2007 to 2017. Data were obtained from the hospital information system at DATASUS. Results: In the analyzed period, 342.139 children aged $\leq 1$ year were hospitalized as a result of respiratory diseases, and 3.062 of these hospitalizations were due to pertussis. In the pre-vaccination period, the annual average was
\end{abstract}

Trabalho realizado na Universidade do Extremo Sul Catarinense (UNESC) - Criciúma (SC), Brasil.

Correspondência: Sabrina Dimer da Silva. E-mail: sabrina_dimer@hotmail.com

Fonte de financiamento: Nenhuma.

Conflito de interesses: nada a declarar.

Recebido em: Jul. 09, 2019. Aprovado em: Jul. 20, 2020.
Este é um artigo publicado em acesso aberto (Open Access) sob a licença Creative Commons Attribution, que permite uso, distribuição e reprodução em qualquer meio, sem restrições desde que o trabalho original seja corretamente citado. 
$102.2 / 100,000$ and, in the post-vaccination period, it was $46.8 / 100.000$, representing a $54.2 \%$ decrease in the number of records, varying from 27.8/100.000/year in 2007 to 139.0/100.000/year in 2013, and reducing to $47.9 / 100.000 /$ year in 2017. Conclusion: There was a reduction in the number of hospitalizations due to pertussis after the introduction of the DTaP vaccine, even with vaccination coverage $<50 \%$.

Keywords: pertussis vaccine; vaccination coverage; child.

\section{INTRODUÇÃO}

A coqueluche é uma doença infectocontagiosa aguda do trato respiratório causada pela bactéria Bordetella pertussis ${ }^{1-13}$ e se encontra na lista das doenças de notificação compulsória, com grande capacidade de disseminação do agente causador, exigindo a aplicação de medidas eficazes de prevenção e controle rigoroso $0^{14-22}$.

Esse agravo está entre as doenças bacterianas de maior incidência em vários países, incluindo o Brasil, com impacto na morbimortalidade, principalmente, de crianças menores de 1 ano de idade em razão de ainda não terem recebido o esquema completo de vacinação ${ }^{1-3,6,7,9,11,12}$. Apesar dos esforços despendidos pelo Programa Nacional de Imunização (PNI) e outros centros de vigilância internacionais, no mundo, em 2012, foi estimada a ocorrência de 50 milhões de casos, com 300 mil óbitos registrados anualmente ${ }^{4,5,9}$. Nesse mesmo período, a coqueluche foi considerada a quinta causa de morte no mundo em menores de 5 anos ${ }^{5-8}$.

Diante disso, o Ministério da Saúde disponibiliza, desde novembro de 2014, no Calendário Nacional pelo Sistema Único de Saúde (SUS), a vacina dTpa (vacina acelular contra difteria, tétano e coqueluche) para gestantes ${ }^{14,22}$. A recomendação é a aplicação da dose na $20^{a}$ semana de gestação, podendo ser administrada até 20 dias antes da data provável do parto e também no puerpério, tendo uma efetividade estimada de $91 \%^{5,14,22}$. O objetivo é transferir, por via transplacentária, os anticorpos da vacina para mãe e, consequentemente, proteger o recémnascido até receber sua primeira dose da vacina ${ }^{1-5,14,22}$.

Desse modo, o objetivo do presente estudo foi analisar o comportamento dos registros de hospitalizações por coqueluche em crianças menores de 1 ano de idade, conforme a cobertura da vacina dTpa para gestantes.

\section{MÉTODO}

Trata-se de um estudo ecológico de caráter observacional e quantitativo. Em outubro de 2018, foi acessada a base de dados do DATASUS que contém informações sobre fatores ambientais, de epidemiologia e morbidade de várias doenças que afetam a saúde da população brasileira. Foram coletadas informações das hospitalizações registradas por doenças respiratórias, não respiratórias e coqueluche na região Sul do país no período de 2007 a 2017.

Para o acesso aos dados de hospitalização, foram utilizados os links de Informações de Saúde (TABNET) $\rightarrow$ Epidemiológicas e Morbidade $\rightarrow$ Morbidade Hospitalar, Lista Morbidade CID10 (Coqueluche - A37.0), do período de 2007 a 2017, com o grupo etário de menores de 1 ano de idade de ambos os sexos ${ }^{7}$.

Para calcular a incidência, foi utilizada a fórmula: $\mathrm{n}^{\circ}$ total de casos confirmados da doença $/ \mathrm{n}^{\circ}$ populacional da faixa etária estudada (por ano e local [Brasil (região Sul) - IBGE]) $\mathrm{x}$ 100.000 habitantes) ${ }^{8}$. Os dados relacionados à vacinação das gestantes foram consultados no Sistema de Informação Hospitalar (SIH) e no Sistema de Informações do Programa Nacional de Imunizações (SI-PNI)9,11. Para avaliar o comportamento dos registros de hospitalizações pré e pós-introdução da vacina dTpa para gestantes, nos casos de coqueluche foi calculada a redução absoluta e relativa simples a partir dos períodos pré (2011-2013) e pós (2015-2017), ou seja, três anos antes e três anos depois de sua implementação. Para essa análise pré e pós-vacinal, 2014 foi considerado o ano de transição para este estudo por ser o período de introdução da vacina e também pela cobertura ser muito baixa $(6,47 \%)$ na região Sul, segundo dados do SIPNI. Contudo, foi avaliado o ano de 2014 mês a mês.

Admissões não respiratórias foram utilizadas como comparação (todos os CID-10, excluindo capítulo X: J00-J99), durante o mesmo período de tempo, além de todas as internações por outras doenças respiratórias. A diferença nas taxas de registros e no índice de 
associação odds ratio (OR) dos períodos pré e pós foi utilizada para avaliar a diferença estatística significativa, considerando um intervalo de confiança (IC) de $95 \%$.

Todos os dados foram revisados pelo autor e por mais dois pesquisadores independentes para garantir sua qualidade. Este estudo apresenta resultados secundários e não contém dados pessoais ou individuais, portanto foi considerado isento de avaliação pelo Comitê de Ética em Pesquisa da Universidade do Extremo Sul Catarinense (UNESC).

Este estudo foi realizado por pesquisadores da UNESC e do Grupo de Pesquisa em Gestão do Cuidado, Integralidade e Educação na Saúde (GECIES). Os dados coletados representam os registros hospitalares por coqueluche citados no DATASUS somente na região Sul do Brasil, abrangendo os 29 municípios do Paraná, 28 do Rio Grande do Sul e 18 de Santa Catarina.

\section{RESULTADOS}

No período estudado, foram registradas 342.139 hospitalizações por doenças respiratórias em crianças menores de 1 ano de idade, sendo que 3.062 foram por coqueluche, no Sul do Brasil.

A distribuição anual demonstra um aumento no número de registros por coqueluche, com 36,2/100.000/ano, 131,5/100.000/ano e 139/100.000/ano, em 2011, 2012 e 2013, respectivamente, chegando a 2014 com uma taxa de hospitalizações de 109,2/100.000/ano - nesta pesquisa, o ano de 2014 foi considerado como o período de transição para o início da aplicação da vacina dTpa para gestantes (Tabela 1).

O número de registros em 2015 sofreu um declínio de 42,6\% em relação ao ano anterior. O aumento do número de registros hospitalares acompanhou o aumento global de notificações nos anos de 2011 a 2014, demonstrando tendência de queda no período de 2015 a 2017 (Figura 1).

No período pré-vacinal (2011-2013), a média anual de hospitalizações por coqueluche em crianças menores de 1 ano de idade foi de 102,2/100.000/ano e, no período pós-vacinal (2015-2017), foi de 46,8/100000/ano, representando uma queda de $54,2 \%$ no número de registros, variando de 27,8/100.000/ano em 2007 a 139/100.000/ano em 2013, caindo para 47,9/100.000/ano em 2017. Quando comparados os registros hospitalares por doenças não respiratórias na mesma unidade de tempo (pré e pós-introdução da vacina) e faixa etária, não foram observadas diferenças significativas (Tabela 2).

A partir do ano de 2014, o PNI disponibilizou a vacina dTpa para gestantes. Em 2015, na região Sul do país, o Programa atingiu $35,6 \%$ de cobertura, coincidindo com a queda de $54,2 \%$ na média anual de incidência (Figura 2).

Tabela 1. Hospitalizações por coqueluche e doenças não respiratórias no Sul do Brasil de 2007 a 2017 em crianças menores de 1 ano de idade

\begin{tabular}{ccc} 
Ano & $\begin{array}{c}\text { Hospitalizações por coqueluche } \\
\text { (incidência por 100.000 } \\
\text { habitantes) }\end{array}$ & $\begin{array}{c}\text { Hospitalização por doenças não } \\
\text { respiratórias (incidência por } \\
\mathbf{1 0 0 . 0 0 0} \text { habitantes) }\end{array}$ \\
\hline 2007 & $145(27.8)$ & $52.819(10130.26)$ \\
\hline 2008 & $213(41.6)$ & $53.594(10461.92)$ \\
\hline 2009 & $142(28.2)$ & $52.886(10495.66)$ \\
\hline 2010 & $67(13.5)$ & $53.545(10791.00)$ \\
\hline 2011 & $177(36.2)$ & $54.249(11089.22)$ \\
\hline 2012 & $635(131.5)$ & $54.397(11265.89)$ \\
\hline 2013 & $663(139.0)$ & $54.563(11437.66)$ \\
\hline 2014 & $515(109.2)$ & $58.127(12322.45)$ \\
\hline 2015 & $292(62.6)$ & $60.666(12997.75)$ \\
\hline 2016 & $139(30.1)$ & $62.010(13420.08)$ \\
\hline 2017 & $219(47.9)$ & $63.926(13968.70)$ \\
\hline
\end{tabular}

Dados apresentados em frequência absoluta e relativa. Fonte: DATASUS (2018) ${ }^{22}$ 
Tabela 2. Hospitalizações por coqueluche e doenças não respiratórias em crianças menores de 1 ano de idade no período pré-vacina (2011-2013) e pós-vacina (2015-2017) no Sul do Brasil, de acordo com o DATASUS

\begin{tabular}{|c|c|c|c|c|c|}
\hline \multirow{2}{*}{ Idade } & \multirow{2}{*}{\multicolumn{2}{|c|}{$\begin{array}{c}2011-2013 \text { (pré) } \quad 2015-2017 \text { (pós) } \\
\begin{array}{c}\text { No de hospitalizações (incidência } \\
\text { por } 100.000 / \text { habitantes/ano) }\end{array}\end{array}$}} & \multirow{2}{*}{$\begin{array}{c}\text { Diferença } \\
\text { absoluta } \\
\text { incidência (pré- } \\
\text { pós) }\end{array}$} & \multicolumn{2}{|c|}{ Diferença relativa incidência (pré-pós) } \\
\hline & & & & $\%$ & OR (IC) \\
\hline \multicolumn{6}{|c|}{ Coqueluche $<1$ ano } \\
\hline Média & $491.6(102.2)$ & $216.6(46.8)$ & -55.4 & -54.2 & $0.45(0.32-0.64) *$ \\
\hline Total & $1.475(306.7)$ & $650(140.6)$ & -166.1 & -54.1 & $0.45(0.37-0.55)^{*}$ \\
\hline \multicolumn{6}{|c|}{ Controle $<1$ ano } \\
\hline Média & $54.403(112.5)$ & $62.200(134.5)$ & 22 & 16.4 & $1.19(0.93-1.53)$ \\
\hline Total & $163.209(337.7)$ & $186.602(403.7)$ & 66 & 16.3 & $1.19(1.03-1.38)$ \\
\hline
\end{tabular}

Dados apresentados em frequência absoluta e relativa. Fonte: DATASUS (2018) ${ }^{22 . *}$ Diferença significativa

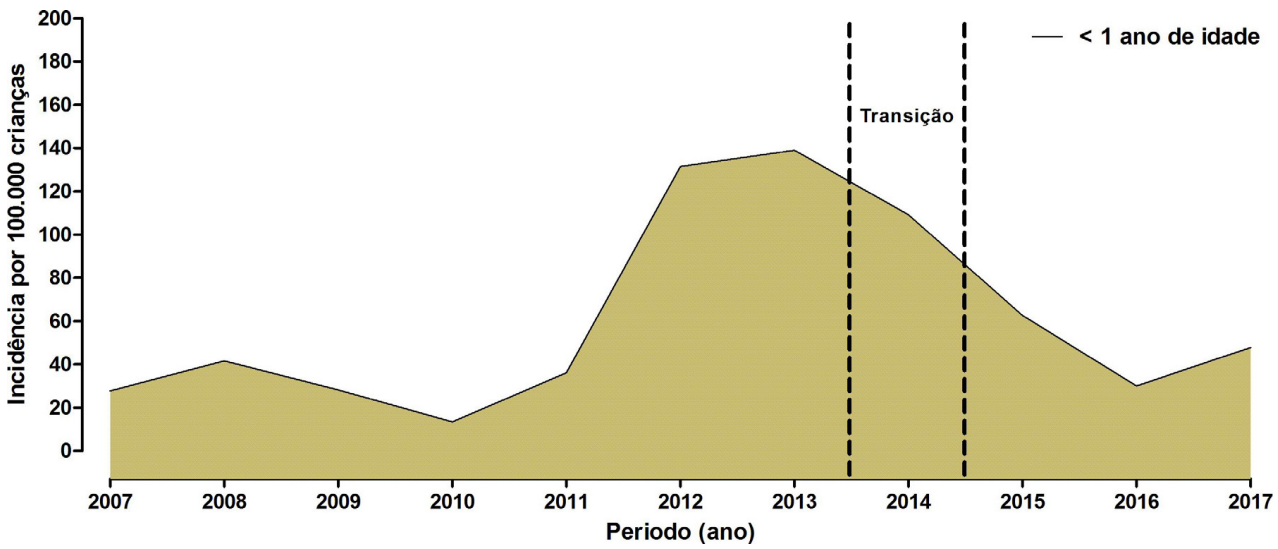

Figura 1. Incidência de hospitalizações por coqueluche de 2007 a 2017 em crianças menores de 1 ano de idade no Sul do Brasil. Fonte: DATASUS (2018) ${ }^{22}$

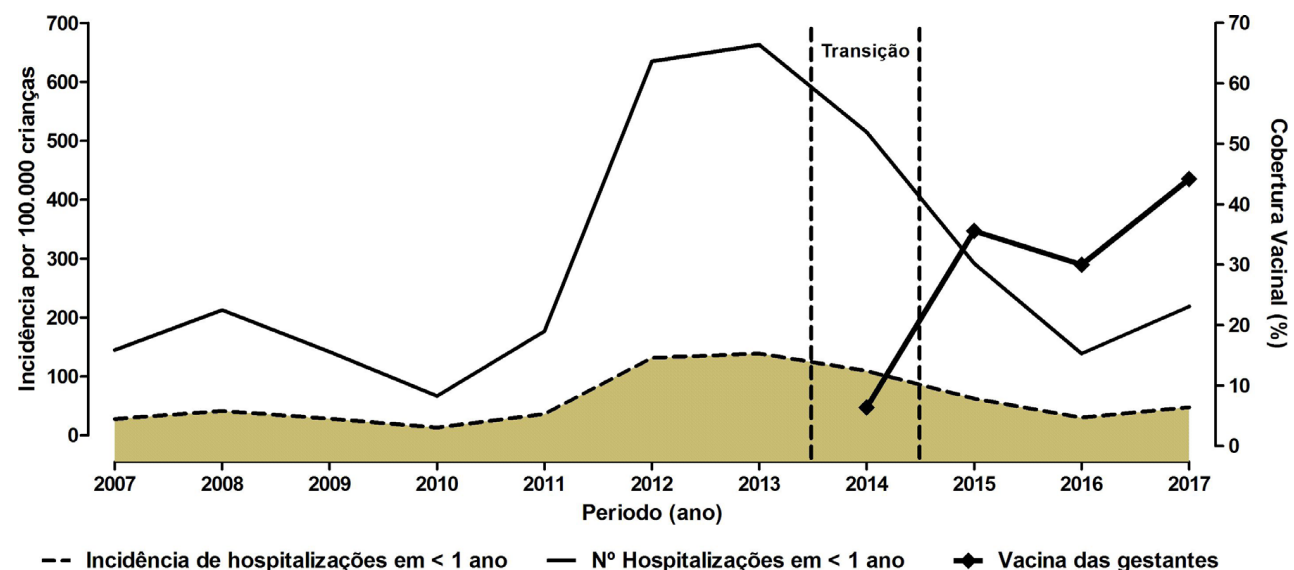

Figura 2. Hospitalizações por coqueluche de 2007 a 2017 em crianças menores de 1 ano de idade e cobertura vacinal das gestantes no Sul do Brasil. Fonte: DATASUS (2018) 22

Em uma análise mês a mês para o ano de 2014, foi observado um maior pico de incidência em fevereiro, com 28,4/100.000/mês; já em dezembro caiu para 11,5/100.000/mês, coincidindo com a introdução da vacina no mês de novembro com uma cobertura de $6,47 \%$ para região Sul do país (Figura 3). 


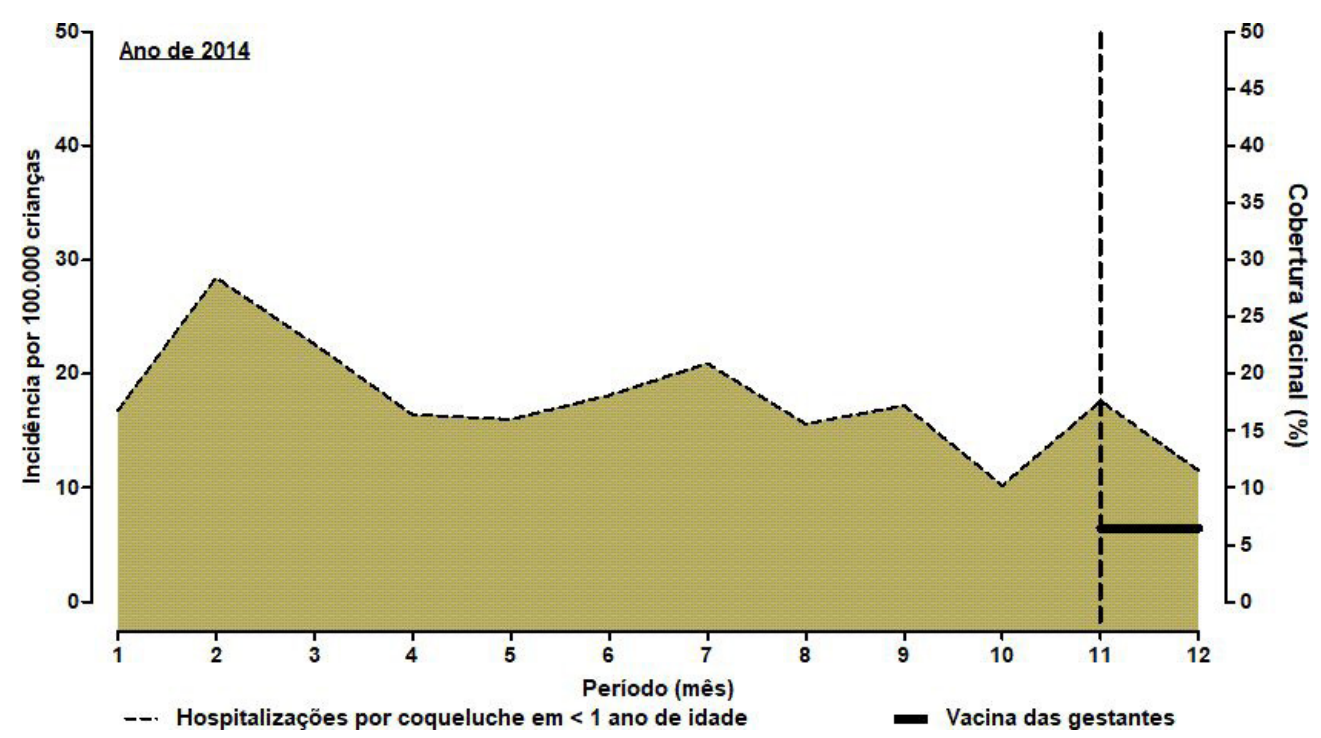

Figura 3. Incidência de hospitalizações por coqueluche mês a mês para o ano de 2014 em crianças menores de 1 ano de idade e cobertura vacinal das gestantes no Sul do Brasil. Fonte: DATASUS (2019) 22

\section{DISCUSSÃO}

Este estudo demonstrou que, após a introdução da vacina dTpa para gestantes, houve uma redução no número de registros de hospitalizações por coqueluche, mesmo com a cobertura vacinal abaixo de $50 \%$.

Essa redução também foi demonstrada em outros estudos. Em Salvador, Bahia, em 2014, a incidência foi de 188/100.000. Já em 2015, as taxas apresentaram uma diminuição importante para 75/100.000, mantendo essa taxa decrescente em 2016 com 22/100.000 $0^{3,4}$.

Dados do DATASUS, em outubro de 2017, apontaram que, em todo o Brasil, a cobertura vacinal contra coqueluche (dTpa) entre gestantes chegou a apenas $38,4 \%$. O Distrito Federal e o Acre tiveram as menores coberturas em 2016, com apenas 15,8\% e 15,4\%, respectivamente. Comparando com 2015, houve uma queda de 37 p.p. no Distrito Federal, quando a cobertura era de $53 \% \%^{5,9,12-15}$.

A região Sudeste também teve uma das maiores baixas, passando de $53,9 \%$ para $37,2 \%$ entre 2015 e 2017, com destaque para o estado de São Paulo, onde a cobertura vacinal contra a doença caiu 36 p.p. nesses dois anos. Já na região Nordeste, a cobertura caiu de $44,2 \%$ em 2015 para 40,2\% em 2017. Em 2015, na região Sul do país, que foi a estudada neste trabalho, o Programa atingiu 35,6\% de cobertura, aumentando para 43,56\% em 2017 e coincidindo com a queda de $54,2 \%$ na média anual de incidência. Os estados que tiveram maior cobertura vacinal foram Ceará (73\%) e Rio de Janeiro (64\%) $)^{5,9,12-15}$.

No cenário mundial, na Inglaterra, no ano de 2012, com a implementação do programa de vacinação das gestantes contra pertussis e a partir de casos confirmados por critérios laboratoriais, foi possível analisar a eficácia da vacina ${ }^{13-22}$. O estudo incluiu 26.684 mulheres que deram à luz a recém-nascidos vivos de outubro de 2012 a setembro de 2013. Os primeiros nove meses de 2013 demonstraram uma queda de $78 \%$ no número de casos confirmados e de $68 \%$ nas hospitalizações por coqueluche ${ }^{16}$. Estudos no Reino Unido apontaram que, em curto prazo, a introdução da vacina materna reduziu consideravelmente a morbimortalidade e apresentou uma eficácia de $90 \%$, além de ser segura para mãe e filho ${ }^{23,24}$.

O presente estudo apresenta algumas limitações relacionadas aos dados de hospitalização do $\mathrm{SIH}$, como qualidade do banco de dados, especificidades de pagamento e uso exclusivo do SUS.

A divulgação dos dados existentes pode contribuir para novas investigações visando explorar outros fatores que podem interferir no número de registros por coqueluche. Assim, é interessante um acompanhamento por um período de tempo maior para avaliar períodos cíclicos da doença, bem como o aumento da cobertura vacinal para gestantes. 


\section{REFERÊNCIAS}

1. Verçosa RCM, Pereira TS. Impacto da vacinação contra pertussis sobre os casos de coqueluche. Rev Enferm UFPE on line. 2017;11(9):3410-8. http://dx.doi.org/10.5205/reuol.11088-99027-5-ED.1109201712.

2. Brasil. Ministério da Saúde. Secretaria de Vigilância em Saúde. Departamento de Vigilância Epidemiológica. Doenças infecciosas e parasitárias; guia de bolso [Internet]. Brasília: Ministério da Saúde; 2010 [citado em 2016 jan 11].Disponível em:http://bvsms.saude.gov.br/bvs/publicacoes/doencas_infecciosas_parasitaria_ guia_bolso.pdf

3. Paixão CSF. Epidemiologia da coqueluche no municipio de salvador-ba no período de 2007 a 2016 [dissertação]. São Paulo: Faculdade de Ciências Médicas da Santa Casa de São Paulo; 2018.

4. Korppi M. Coqueluche: ainda um desafio. J Pediatr. 2013;89(6):520-2. http://dx.doi.org/10.1016/j. jped.2013.09.001.

5. World Health Organization. Immunization, vaccines and biologicals: pertussis [Internet]. Geneva: WHO 2011 [citado em 2016 jan 28]. Disponível em: http://www.who.int/immunization/topics/pertussis/en/

6. Brasil. Ministério da Saúde. Comissão Nacional de Incorporação de Tecnologias do SUS . Relatório n 46 , Portaria n 47, de 10 de setembro de 2013 [Internet]. Brasília: Ministério da Saúde; 2013 [citado em 2016 ago 17]. Disponível em: http://bvsms.saude.gov.br/bvs/saudelegis/sctie/2013

7. Brasil. Ministério da Saúde. Tabnet DATASUS. Indicadores básicos para a saúde no Brasil [Internet]. Brasília: Ministério da Saúde; 2013 [citado em 2016 ago 17]. Disponível em: http://tabnet.datasus.gov.br

8. Instituto Brasileiro de Geografia e Estatística [Internet]. 2016 [citado em 2017 jan 17]. Disponível em: http://www.ibge.gov.br

9. Brasil. Ministério da Saúde. Secretaria de Vigilância em Saúde. Programa Nacional de Imunizações (PNI): 40 anos [Internet]. Brasília: Ministério da Saúde; 2013 [citado em 2016 jan 12]. Disponível em: http:// bvsms.saude.gov.br/bvs/publicacoes/programa_nacional_imunizacoes_pni40.pdf

10. Brasil. Ministério da Saúde. Sistema de Informação de Agravos de Notificação - SINAN [Internet]. Brasília: Ministério da Saúde; 2016 [citado em 2016 abr 10]. Disponível em: http://sinan.saude.gov.br

11. Brasil. Ministério da Saúde. Coordenação geral do programa de imunização. Informe técnico para implantação da vacina adsorvida difteria, tétano e coqueluche (pertussis acelular) tipo adulto - DTPA [Internet]. Brasília: Ministério da Saúde; 2014 [citado em 2016 abr 10]. Disponível em: http://www.crmpr. org.br/uploadAddress/info_dTpa_ministerio-saude-setembro-2014\%5B1614\%5D.pdf

12. Correia CA. Influência da vacinação com dTpa em gestantes no perfil da resposta imunológica contra a Bordetella pertussis na criança [dissertação]. São Paulo: Instituto de Medicina Tropical, Universidade de São Paulo; 2018

13. Guimarães LM, Carneiro ELNC, Carvalho-Costa FA. Increasing incidence of pertussis in Brazil: a retrospective study using surveillance data. BMC Infect Dis. 2015;15(1):442-54. http://dx.doi.org/10.1186/s12879-0151222-3. PMid:26498058.

14. Willemann MCA, Goes FCS, Araújo ACM, Domingues CMAS. Adoecimento por coqueluche e número de doses administradas de vacinas Pertussis: estudo de caso controle. Epidemiol Serv Saude. 2014;23(2):20714. http://dx.doi.org/10.5123/S1679-49742014000200002.

15. Locht $\mathrm{C}$, Mielcarek N. New pertussis vaccination approaches: en route to protect newborns? FEMS Immunol Med Microbiol. 2012 nov;66(2):121-33. http://dx.doi.org/10.1111/j.1574-695X.2012.00988.x. PMid:22574832.

16. Amirthalingam G, Andrews N, Campbell H, Ribeiro S, Kara E, Donegan K, et al. Effectiveness of maternal pertussis vaccination in England: an observational study. Lancet. 2014;384(9953):1521-8. http://dx.doi. org/10.1016/S0140-6736(14)60686-3. PMid:25037990.

17. Torres RSLA, Santos T, Torres RAA, Pereira VG, Fávero LAF, Filho OR, et al. Resurgence of pertussis at the age of vaccination: clinical, epidemiological, and molecular aspects. J Pediatr. 2015;91(4):333-8. http:// dx.doi.org/10.1016/j.jped.2014.09.004. PMid:25623040.

18. Center for Disease Control and Prevention. Perssis cases by year 1922-2015. Pertussis (Whooping) [Internet]. 2017 [citado em 11 out 2017]. Disponível em: httpa://www.cdc.gov/pertussis/surv-reporting cases-by-year.html

19. Centers for Disease Control and Prevention. Updated recommendations for use of tetanus toxoid, reduced diphtheria toxoid, and acellular pertussis vaccine (Tdap) in pregnant women. MMWR Morb Mortal Wkly Rep. 2013;62(7):131-5. PMid:23425962.

20. Kretsinger K, Broder KR, Cortese MM, Joyce MP, Ortega-Sanchez I, Lee GM, et al. Preventing tetanus, diphtheria, and pertussis among adults: use of tetanus toxoid, reduced diphtheria toxoid and acellular pertussis vaccine. MMWR Recomm Rep. 2006;55(RR-17):1-37. PMid:17167397. 
21. Baptista PN, Magalhães VS, Rodrigues LC. The role of adults in household outbreaks of pertussis. Int J Infect Dis. 2010;14:e111-4. http://dx.doi.org/10.1016/j.ijid.2009.03.026.

22. Brasil. Ministério da Saúde. Departamento de Informática do SUS DATASUS 2008-2014 [Internet]. Brasília: Ministério da Saúde; 2016 [citado em 2016 maio 21]. Disponível em: http://www2.datasus.gov.br/ DATASUS/index.php

23. Organização Mundial da Saúde. Recomendações da OMS sobre cuidados pré-natais para uma experiência positiva na gravidez [Internet]. Geneva: OMS; 2016. p. 1-10 [citado em 2017 dez 12]. Disponível em: http:// apps.who.int/iris/bitstream/10665/250800/2/WHO-RHR-16.12-por.pdf

24. Vizzotti C, Juarez MV, Bergel E, Romanin V, Califano G, Sagradini S, et al. Impact of a maternal immunization program against pertussis in a developing country. Vaccine. 2016;34(50):6223-8. http://dx.doi. org/10.1016/j.vaccine.2016.10.081. PMid:27847175. 